\title{
Influence of Fibers on the Flow Through the Hot-End in Material Extrusion Additive Manufacturing
}

Serdeczny, Marcin; Comminal, Raphaël; Pedersen, David Bue; Spangenberg, Jon

Published in:

Industrializing Additive Manufacturing

Link to article, DOI:

10.1007/978-3-030-54334-1_18

Publication date:

2020

Document Version

Peer reviewed version

Link back to DTU Orbit

Citation (APA):

Serdeczny, M., Comminal, R., Pedersen, D. B., \& Spangenberg, J. (2020). Influence of Fibers on the Flow Through the Hot-End in Material Extrusion Additive Manufacturing. In M. Meboldt, \& C. Klahn (Eds.), Industrializing Additive Manufacturing: Proceedings of AMPA 2020 (pp. 251-267). Springer. https://doi.org/10.1007/978-3-030-54334-1_18

\section{General rights}

Copyright and moral rights for the publications made accessible in the public portal are retained by the authors and/or other copyright owners and it is a condition of accessing publications that users recognise and abide by the legal requirements associated with these rights.

- Users may download and print one copy of any publication from the public portal for the purpose of private study or research.

- You may not further distribute the material or use it for any profit-making activity or commercial gain

- You may freely distribute the URL identifying the publication in the public portal 


\title{
Influence of fibers on the flow through the hot-end in material extrusion additive manufacturing
}

\author{
Marcin P. Serdeczny, Raphaël Comminal, David B. Pedersen, Jon Spangenberg ${ }^{1}$ \\ Technical University of Denmark, Department of Mechanical Engineering, \\ 2800 Kgs.Lyngby, Denmark \\ 1josp@mek.dtu.dk
}

\begin{abstract}
Advancements in material extrusion additive manufacturing are driven by the need of fast production of high-quality parts. A key innovation was the introduction of short fibers into the built filament, which substantially improve the strength of manufactured components. However, the presence of fibers also affects the viscosity and thermal conductivity of the filament, which are important parameters for the extrusion flow and thus the maximum printing speed. In this paper, we numerically study the effect of fibers on the polymer flow and pressure drop inside the nozzle, which determines the maximum extrusion rate. The thermoplastic polymer flow is simulated with a non-isothermal computational fluid dynamics model and the inclusion of fibers is treated with a continuum approach. The simulations are performed for ABS polymer with short carbon fibers, however, other thermoplastic systems with short fibers (e.g. glass, wood, or nylon) can be integrated into the model. The model provides a virtual window into the process and illustrates that while fibers increase the viscosity of the filament and the pressure drop, they also improve the thermal conductivity and lead to faster melting of the polymer, which has an opposite effect on the pressure drop. Finally, the model quantifies the relationship between the fiber volume fraction and the maximum extrusion rate.
\end{abstract}

Keywords: Fused Deposition Modeling, Fiber Reinforcement, Computational Fluid Dynamics.

\section{Introduction}

Material extrusion Additive Manufacturing (AM), or Fused Deposition Modeling (FDM), is currently undergoing a transition from being a prototyping technique to producing functional parts with industrial-grade mechanical strength. One of the key drivers behind this progress is the inclusion of fiber reinforcement inside the filament material, which improves the mechanical performance of the produced components [1]. Short carbon fibers are a popular type of reinforcement used in material extrusion AM $[2,3]$. In addition to increasing the mechanical strength, the carbon fibers also improve the thermal conductivity and reduce the coefficient of thermal expansion of the compound material, which leads to less thermal warpage and lower residual stresses $[4,5]$. Functional parts made by carbon reinforced material extrusion AM can potentially be 
applied in medical equipment [6], aerospace [7-9], automotive [10] and consumer products [11].

Experimental studies have been conducted to quantify the improvement in tensile strength and elastic modulus of 3D printed components, due to the presence of short carbon fibers [1, 3]. Computational Fluid Dynamics (CFD) simulations have been widely used to model the polymer flow during material extrusion AM [12-18], among which the works by Heller et al. $[19,20]$ focused on the prediction of the fiber orientation in the extrusion and deposition flow. Thermo-mechanical simulations were conducted to predict the deformations and residual stresses in the finished carbon/polymer composites [21, 22]. However, there is a limited research on the influence of fiber reinforcement on the melting and flow of polymer composite inside the print head. The addition of fibers alters the rheology and the thermal properties of the fluid, which are both highly important for the flow behavior.

Heat transfer effects and rheology are kesy aspects to increasing the build rates in material extrusion AM [23]. Mackay et al. [24] determined the failure velocity that was defined as the filament feeding rate at which the heater was not able to melt efficiently the polymer to perform successful extrusion. Nienhaus et al. [25] used a load cell to measure the dependence of the filament feeding force on the feeding rate. They found that the feeding force becomes unstable before reaching the failure velocity. This observation was confirmed by Serdeczny et al. [26], where a model to estimate the maximum feeding rate before the extrusion becomes unstable was presented. It was hypothesized that the maximum feeding rate is limited by the heat transfer and the ability to melt the filament completely before it reaches the nozzle contraction. A more detailed explanation of the extrusion instability was given in a follow-up work [27] presenting numerical simulations of the flow inside the print head. The feeding force instability was hypothesized to be due to a fluctuation of the melt zone position inside the flow channel.

In this work, we modify the CFD model presented in [27] to investigate the influence of fiber additives on the flow inside the printing head and the melting of filament. The inclusion of fibers is simulated with a continuum approach by modifying the viscosity and thermal conductivity of the fluid. A parametric study on the influence of the fiber content and feeding rate is performed.

\section{Methodology}

\subsection{Physics of the numerical model}

The numerical model simulates the polymer flow inside the hot-end channel of a 3D printer. A sketch representing the hot-end is shown in Fig. 1a. The essential elements of the hot-end are: the nozzle that extrudes the material, the liquefier that melts the polymer, the heat break that isolates thermally the liquefier from the upper parts of the print head, and the heat sink, which releases the excess heat to the environment. In the numerical model, the internal channel of the hot-end was implemented, as shown in Fig. 1b. The filament entered the channel with a constant velocity $V$, termed the feeding 
rate and the inlet temperature $T_{I N}$, equal to the room temperature. The material remained solid until its temperature reached the glass temperature of the polymer $T_{g}$. The channel was subdivided into five sections with dimensions as indicated in the Figure $1 \mathrm{~b}$. It is noted that the filament had a slightly smaller diameter $D_{F}$ than the barrel section $D_{B}$. It was assumed that the wall of the channel had a steady temperature, which varies along the channel, as shown in Fig. 1c. The geometry of the channel was based on the E3D v6 hot-end and its dimensions are given in Appendix A.

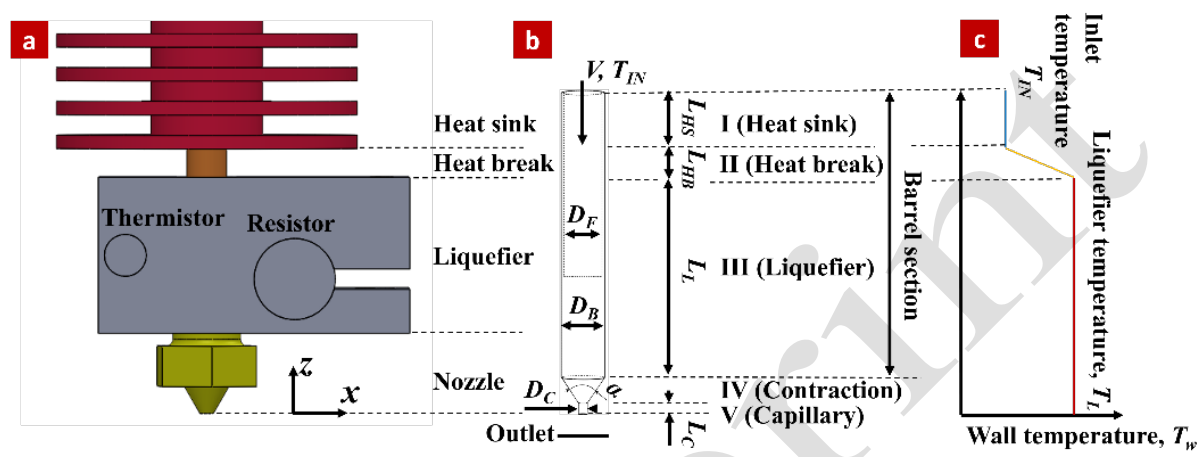

Fig. 1. a) Sketch of the hot-end. b) Internal channel of the hot-end. c) Wall temperature of the channel.

The flow and temperature of material is governed by the following equations [28]

$$
\begin{gathered}
\nabla \cdot \boldsymbol{u}=0 \\
\rho \frac{D \boldsymbol{u}}{D t}=-\nabla p+\nabla \cdot\left(\eta_{\phi} \overline{\overline{\boldsymbol{S}}}\right)+\rho \boldsymbol{g}_{\boldsymbol{a}} \\
\rho C_{v} \frac{D T}{D t}=\nabla \cdot\left(\lambda_{\phi} \nabla T\right) \\
\overline{\overline{\boldsymbol{S}}}=\nabla \boldsymbol{u}+\nabla \boldsymbol{u}^{\mathrm{T}}
\end{gathered}
$$

where $\boldsymbol{u}$ is the velocity vector, $\rho$ is the density, $t$ is the time, $p$ is the pressure, $\overline{\overline{\boldsymbol{S}}}$ is the strain rate tensor, $\boldsymbol{g}_{\boldsymbol{a}}$ is the gravity acceleration vector, $C_{v}$ is the specific heat capacity, $T$ is the temperature, $\lambda_{\phi}$ is the effective thermal conductivity, and $\eta_{\phi}$ is the apparent dynamic viscosity. Thus, it was assumed that the flow is incompressible and the fluid had a constant density. The fiber suspension in the polymer was approximated with a continuum approach and the flow of only one phase was simulated. However, the free surface of the fluid was resolved and the remaining part of the domain was filled with void that had a constant pressure and temperature. The presence of fibers was accounted in the formula of the apparent fluid viscosity and thermal conductivity, which are discussed in detail in Sections 2.3 and 2.4. The influence of fibers on the fluid density and specific heat was assumed negligible for the flow inside the print head, and their values are given in Table 1 together with the remaining simulation parameters. 
Table 1. Simulation parameters.

\begin{tabular}{lccl}
\hline Parameter & Symbol & Unit & Value \\
\hline Fluid density & $\rho$ & $\mathrm{kg} / \mathrm{m}^{3}$ & 1060 \\
Specific heat capacity & $C_{v}$ & $\mathrm{~J} /(\mathrm{kg} \cdot \mathrm{K})$ & 1750 \\
Inlet temperature & $T_{I N}$ & ${ }^{\circ} \mathrm{C}$ & 25 \\
Glass transition temperature & $T_{g}$ & ${ }^{\circ} \mathrm{C}$ & 105 \\
Liquefier temperature & $T_{L}$ & ${ }^{\circ} \mathrm{C}$ & 200 \\
Feeding rates & $V$ & $\mathrm{~mm} / \mathrm{min}$ & $40,60,80, \ldots, 280$ \\
\hline
\end{tabular}

\subsection{Implementation details}

The numerical simulations were performed using the commercial CFD software Flow3D [29]. The implicit coupled pressure-velocity solver was used to solve the governing equations for the material flow. The energy equation was solved with an implicit timediscretization scheme that is second order accurate in space. The problem was assumed to be axisymmetric and a 2D mesh was constructed as represented in Fig. 2. The average cell size was $20 \mu \mathrm{m}$ and the mesh was refined near the channel walls. At the top of the domain, the velocity inlet boundary condition was prescribed. The continuative boundary, which specifies all spatial derivatives of the fluid properties equal to zero, was used at the outlet located outside of the nozzle orifice. The wall of the channel had a prescribed temperature, as shown in Fig. 1c. The heat flux between the channel wall and the fluid was calculated as

$$
\dot{q}_{w}=h\left(T_{w}-T\right)
$$

where $h$ is the heat transfer coefficient at the channel wall while $T_{w}$ is the channel wall temperature. The value of $h$ is unknown and was found through comparison with experimental measurements of the filament feeding force during extrusion of neat ABS. The measurements and their results are discussed in detail in [26]. It was found that the simulation represents the measurements most closely, when $h \approx 600 \mathrm{~W} /\left(\mathrm{m}^{2} \mathrm{~K}\right)$ in the fully developed flow region, and $h \approx 8000 \mathrm{~W} /\left(\mathrm{m}^{2} \mathrm{~K}\right)$ close to the inlet of the channel where a recirculation of the flow occurs. The simulations were run until a steady state was reached, or for $60 \mathrm{~s}$, when the flow was inherently unsteady. A more in-depth discussion about the implementation of the numerical model can be found in [27]. 


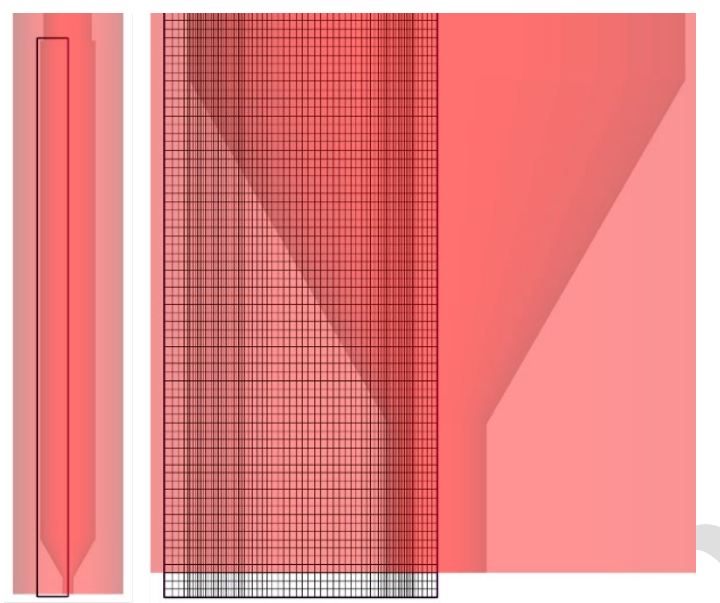

Fig. 2. 2D axisymmetric cylindrical mesh used in the study. Left: outline of the entire mesh. Right: close-up on the contraction section.

\subsection{Rheological properties of the polymer with suspended fibers}

The polymer used in this study is ABS, which is one of the most commonly used thermoplastics in material extrusion AM. The apparent dynamic viscosity of a neat ABS can be described with a temperature dependent power law

$$
\eta(\dot{\gamma}, T)=K \dot{\gamma}^{n-1} \cdot \frac{T}{T_{r}} a_{T}^{n}
$$

where $K$ is the consistency index, $n$ is the power law index, $\dot{\gamma}$ is the shear rate magnitude, $T$ is the polymer temperature, $T_{r}$ is the reference temperature, and $a_{T}$ is the time temperature shift factor. The temperature dependency of $a_{T}$ is modeled with the Williams-Landel-Ferry (WLF) equation

$$
a_{T}(T)=\exp \left(\frac{-C_{1}\left(T-T_{r}\right)}{C_{2}+\left(T-T_{r}\right)}\right)
$$

where $C_{1}$ and $C_{2}$ are material constants. The inclusion of fibers changes the apparent viscosity of the fluid. One of the commonly used simple model to capture this dependence is the equation by Maron and Pierce [30,31]

$$
\eta_{r}=\left(1-\frac{\phi}{A}\right)^{-2}
$$

where $\eta_{r}$ is the relative viscosity, which is defined as the ratio of filled system viscosity $\eta_{\phi}$ to the neat polymer viscosity $\eta$ at the same shear stress (not the same shear rate); $\phi$ is the fiber volume fraction; $A$ is the parameter related to the aspect ratio of the fibers 
[30]. Taking into account the definition of $\eta_{r}$ and combining it with equation (6), the viscosity of the filled system can be written as

$$
\eta_{\phi}=K \dot{\gamma}_{\phi}^{n-1} \frac{T}{T_{r}} a_{T}^{n} \eta_{r}^{n}
$$

The derivation of Equation (9) is given in Appendix B. The strain rate magnitude relates to the velocity vector in the numerical model as

$$
\dot{\gamma}_{\phi}=\sqrt{\frac{1}{2} \overline{\bar{S}}: \overline{\bar{S}}}
$$

The rheological material parameters used in this study are given in Table 2 . The oscillatory measurements of neat $\mathrm{ABS}$ were done for temperatures ranging from $125^{\circ} \mathrm{C}$ to $250{ }^{\circ} \mathrm{C}$ and a master curve was created at $T_{r}=200^{\circ} \mathrm{C}$. The measurement points and the power law fit are shown in Fig. 3. Moreover, we have estimated the dynamic viscosity for ABS filled with fibers at different volume fractions (the weight fractions are also shown for reference). We have assumed that the fibers have an average aspect ratio $\beta=l_{f} / d_{f}$ equal to 23 , where $l_{f}$ is the fiber length and $d_{f}$ is the fiber diameter. For this aspect ratio, the parameter $A$ in equation (8) equals 0.25 , as given in [30]. It is assumed that the material behaves as a Generalized Newtonian Fluid (GNF), meaning that the Trouton ratio (the ratio of elongational viscosity to shear viscosity) is equal to 3 . A large fiber content may invalidate this assumption, necessitating the use of a viscoelastic constitutive model [31]. In such case, the complexity and computational cost of the numerical simulations would be greatly increased.

Table 2. Rheological material parameters.

\begin{tabular}{lllll}
\hline Parameter & Symbol & Unit & Value & Source \\
\hline Reference temperature & $T_{r}$ & ${ }^{\circ} \mathrm{C}$ & 200 & - \\
Consistency index & $K$ & $\mathrm{~Pa} \cdot \mathrm{s}^{\mathrm{n}}$ & 30104 & Measured \\
Power law index & $n$ & - & 0.24 & Measured \\
WLF constant 1 & $C_{1}$ & - & 8.97 & Measured \\
WLF constant 2 & $C_{2}$ & ${ }^{\circ} \mathrm{C}$ & 155.2 & Measured \\
Average fiber aspect ratio & $\beta$ & - & 23 & {$[30,32]$} \\
Parameter in Eq. (8) & $A$ & - & 0.25 & {$[30]$} \\
Fiber density & $\rho_{f}$ & $\mathrm{~kg} / \mathrm{m}^{3}$ & 1800 & {$[33]$} \\
\hline
\end{tabular}




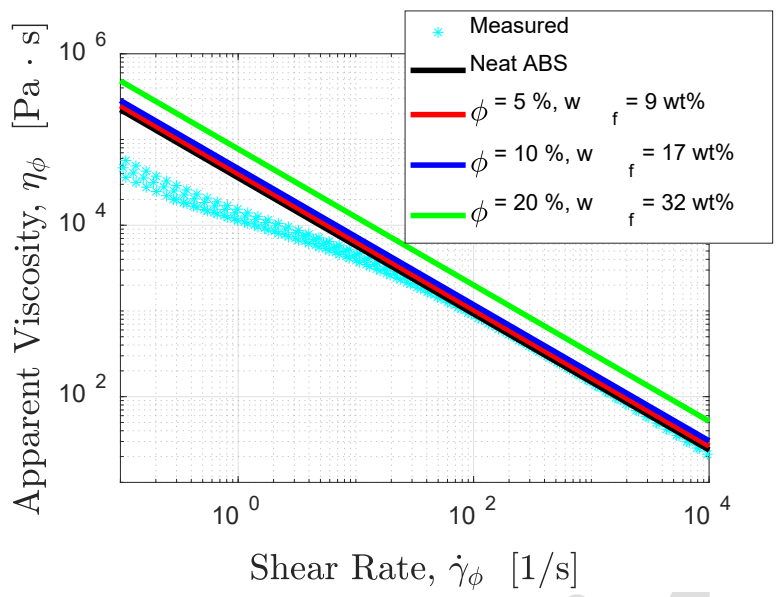

Fig. 3. The master curve for neat $\mathrm{ABS}$ polymer at $T_{r}=200^{\circ} \mathrm{C}$ and estimated dynamic apparent viscosity for fiber suspension with varying fiber volume fraction.

\subsection{Thermal conductivity of the reinforced filaments}

The reinforced filaments used in material extrusion AM are themselves produced by screw extrusion of a matrix (thermoplastic) and fibers compound mixed together. Due to the nature of extrusion compounding, most of the fibers align with the axis of the filament [34-36]. However, there will always exist some deviation of the fiber orientations from the extrusion direction, which can be quantified by a Fiber Orientation Distribution (FOD). Many models to predict the thermal conductivity of fiber reinforced composites were developed, among which we chose to use the approach by Fu and Mai [37], which accounts for the influence of FOD on the thermal conductivity.

A sketch representing the method is shown in Fig. 4. The effective thermal conductivity of a composite consisting of misaligned fibers with known FOD can be calculated as [37]

$$
\lambda_{\phi}=\int_{0^{\circ}}^{180^{\circ}} \lambda(\theta) g(\theta) d \theta
$$

where $\lambda(\theta)$ is the thermal conductivity of a unidirectional fiber composite with its principle axis (' 1 ' longitudinal and ' 2 ' transverse, cf. Fig. 4b) oriented at an angle $\theta$ to the heat transfer direction $x$, and $g(\theta)$ is the FOD function, that is approximated with a normal probability density function, as suggested by experimental observations [35]

$$
g(\theta)=\frac{1}{\sigma_{\theta} \sqrt{2 \pi}} \exp \left(\frac{-\left(\theta-\theta_{\text {mean }}\right)^{2}}{2 \sigma_{\theta}^{2}}\right)
$$

where $\theta_{\text {mean }}$ is the mean fiber orientation angle (that is aligned with the filament axis, i.e. extrusion direction), and $\sigma_{\theta}$ is the standard deviation of the orientation distribution. 
The thermal conductivity of a unidirectional fiber composite at an angle $\theta$ can be calculated as a superposition of its longitudinal thermal conductivity $\lambda_{1}$ (parallel to the fibers) and transverse thermal conductivity $\lambda_{2}$ (perpendicular to the fibers) (cf. Fig. 4b)

$$
\lambda(\theta)=\lambda_{1} \cos ^{2} \theta+\lambda_{2} \sin ^{2} \theta
$$

where the longitudinal and transverse thermal conductivities are calculated using Halpin-Tsai [37] equation, respectively

$$
\begin{gathered}
\lambda_{1}=\frac{1+2 \beta \mu_{1} \phi}{1-\mu_{1} \phi} \lambda_{m} \\
\lambda_{2}=\frac{1+2 \mu_{2} \phi}{1-\mu_{2} \phi} \lambda_{m} \\
\mu_{1}=\frac{\lambda_{f 1} / \lambda_{m}-1}{\lambda_{f 1} / \lambda_{m}+2 \beta} \\
\mu_{2}=\frac{\lambda_{f 2} / \lambda_{m}-1}{\lambda_{f 2} / \lambda_{m}+2}
\end{gathered}
$$

where $\lambda_{m}$ is the thermal conductivity of the polymer matrix, $\lambda_{f 1}$ and $\lambda_{f 2}$ are thermal conductivities of a single fiber in parallel and perpendicular direction to the fiber axis.

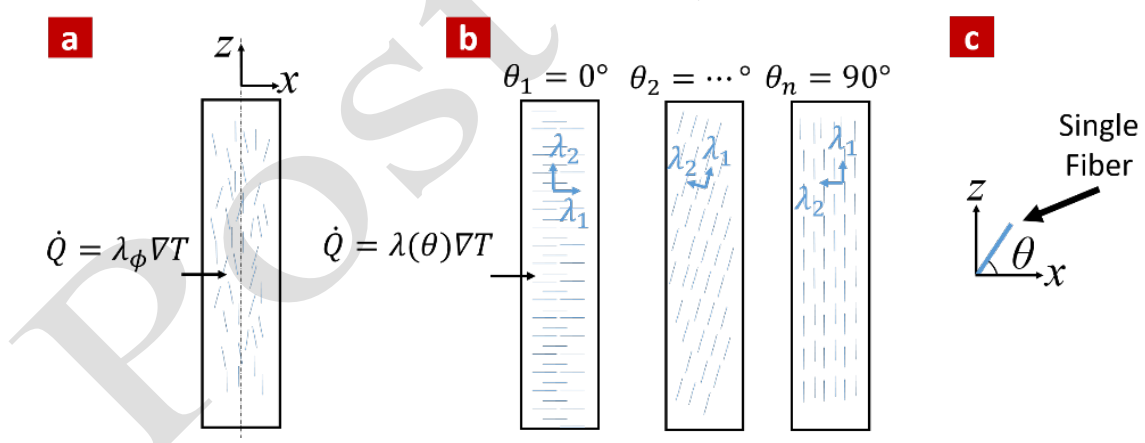

Fig. 4. Sketch representing the thermal conductivity model. a) Filament with misaligned fibers with predominant direction $z$. b) Decomposition into unidirectional fiber composites with fibers aligned at different directions. c) Definition of fiber angle $\boldsymbol{\theta}$.

During the flow through the hot-end, the polymer is heated due to radial conduction from the channel walls while the heat flux due to the axial conduction is usually negligible, as compared to the axial convection. Moreover, as the problem is axisymmetric, $\lambda_{\phi}$ can be assumed to represent the thermal conductivity of the filament in all directions. The parameters used in the estimation of the effective thermal conductivity are 
given in Table 3. The calculated dependence of the effective thermal conductivity on the fiber volume fraction is shown in Fig. 5. For reference, the thermal conductivity of the filament with perfectly aligned fibers $\left(\sigma_{\theta}=0^{\circ}\right)$ is also shown. It is observed that the FOD increases the thermal conductivity and its influence becomes larger with higher volume fractions. In the end, we note that compared to the approach of $\mathrm{Fu}$ and Mai [37], we have not accounted for the fiber length distribution, however its influence on the thermal conductivity was found to be negligible for the present case.

Table 3. Parameters used in the estimation of the effective thermal conductivity of the fiber reinforced filament.

\begin{tabular}{lclll}
\hline Parameter & Symbol & Unit & Value & Source \\
\hline ABS thermal conductivity & $\lambda_{m}$ & $\mathrm{~W} /(\mathrm{m} \cdot \mathrm{K})$ & 0.33 & {$[38]$} \\
Fiber thermal conductivity (parallel) & $\lambda_{f 1}$ & $\mathrm{~W} /(\mathrm{m} \cdot \mathrm{K})$ & 10 & {$[39]$} \\
Fiber thermal conductivity (transverse) & $\lambda_{f 2}$ & $\mathrm{~W} /(\mathrm{m} \cdot \mathrm{K})$ & 10 & {$[39]$} \\
Mean fiber orientation angle & $\theta_{\text {mean }}$ & $\circ$ & 90 & Based on [35] \\
Standard deviation of FOD (Eq. (12)) & $\sigma_{\theta}$ & $\circ$ & 15 & Based on [35] \\
\hline
\end{tabular}

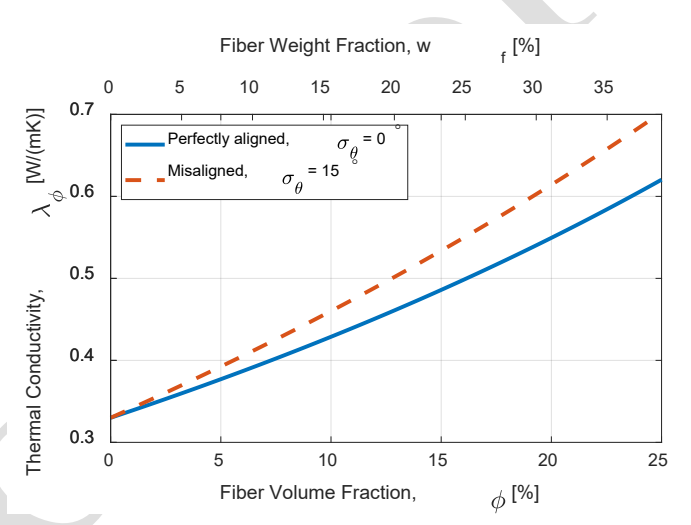

Fig. 5. Influence of the fiber volume fraction and the standard deviation of the fiber orientation on the thermal conductivity in the radial direction of the filament.

\section{Results}

\subsection{Comparison with experimental results}

First, the numerical simulation is validated with experimental measurements of the filament feeding force when a neat $\operatorname{ABS}$ filament $(\phi=0)$ is extruded. The filament feeding force is the force required to push the filament through the hot-end system and it was measured in $[26,40]$ using a load cell, where a detailed description can be found. The comparison of the simulated and measured feeding force as a function of the feeding rate can be seen in Fig. 6. The error bars show the standard deviation of the feeding 
force oscillations during the measurement/simulation of individual data points. It can be seen that for low feeding rates, no oscillations in the feeding force are observed and the extrusion is in the stable regime. The temperature profile within the hot-end for the stable regime can be seen in Fig. 7. At some critical feeding rate, called the maximum feeding rate, the force curve changes slope and oscillations appear, which indicates unstable extrusion. It is noted that these instabilities are distinct from the shark skin and melt fracture extrusion instability, as they occur at a lower frequency and are not related to change in the appearance of the extrudate surface [26]. A similar behavior of the feeding force was measured in [25]. It can be seen that the numerical results are in a good agreement with the measurements and the maximum feeding rate threshold between the stable and unstable regime is captured by the simulations. The unstable regime is related to the insufficient heat transfer rate at large feeding rates. Looking at the evolution of the temperature profile for the unstable regime (cf. Fig. 7), it can be seen that as the heat transfer rate is too small, the solid filament penetrates the channel causing fluctuations in the position of the melt line. The magnitude of the feeding force fluctuations are larger in the simulations than in the experiments. Note that in the simulations, the solid filament below the glass temperature is modelled as a rigid solid, neglecting viscoelastic effects that may damp the pressure variations. Finally, we note that our definition of the maximum feeding rate is based on the extrusion instability coming from the insufficient heat transfer. There are other factors limiting the maximum feeding rate in material extrusion AM, such as the buckling of the filament and the maximum feeding force that can be applied on the filament by the extruder; however their analysis was outside of the scope of this work.

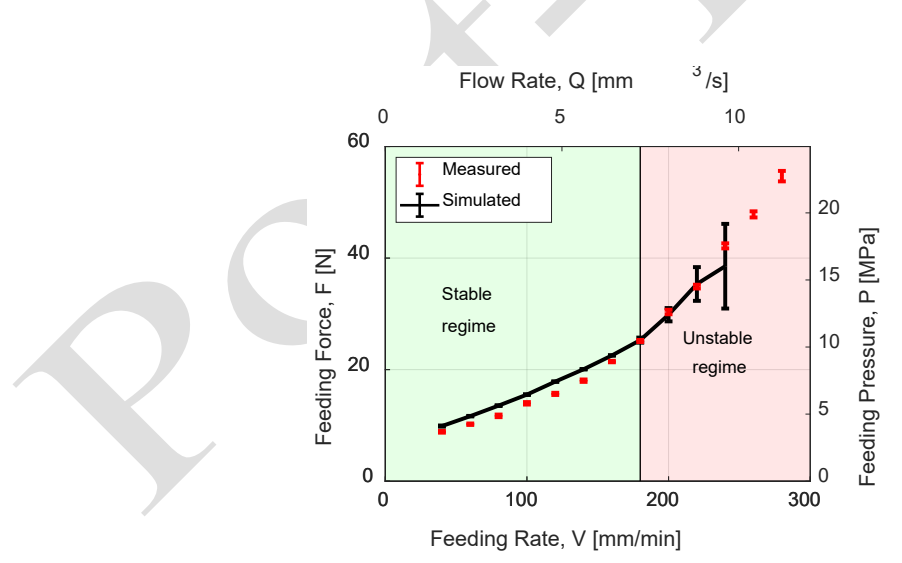

Fig. 6. Comparison of the measured and simulated filament feeding force for the extrusion of neat ABS filament at $T_{L}=200{ }^{\circ} \mathrm{C}$. 


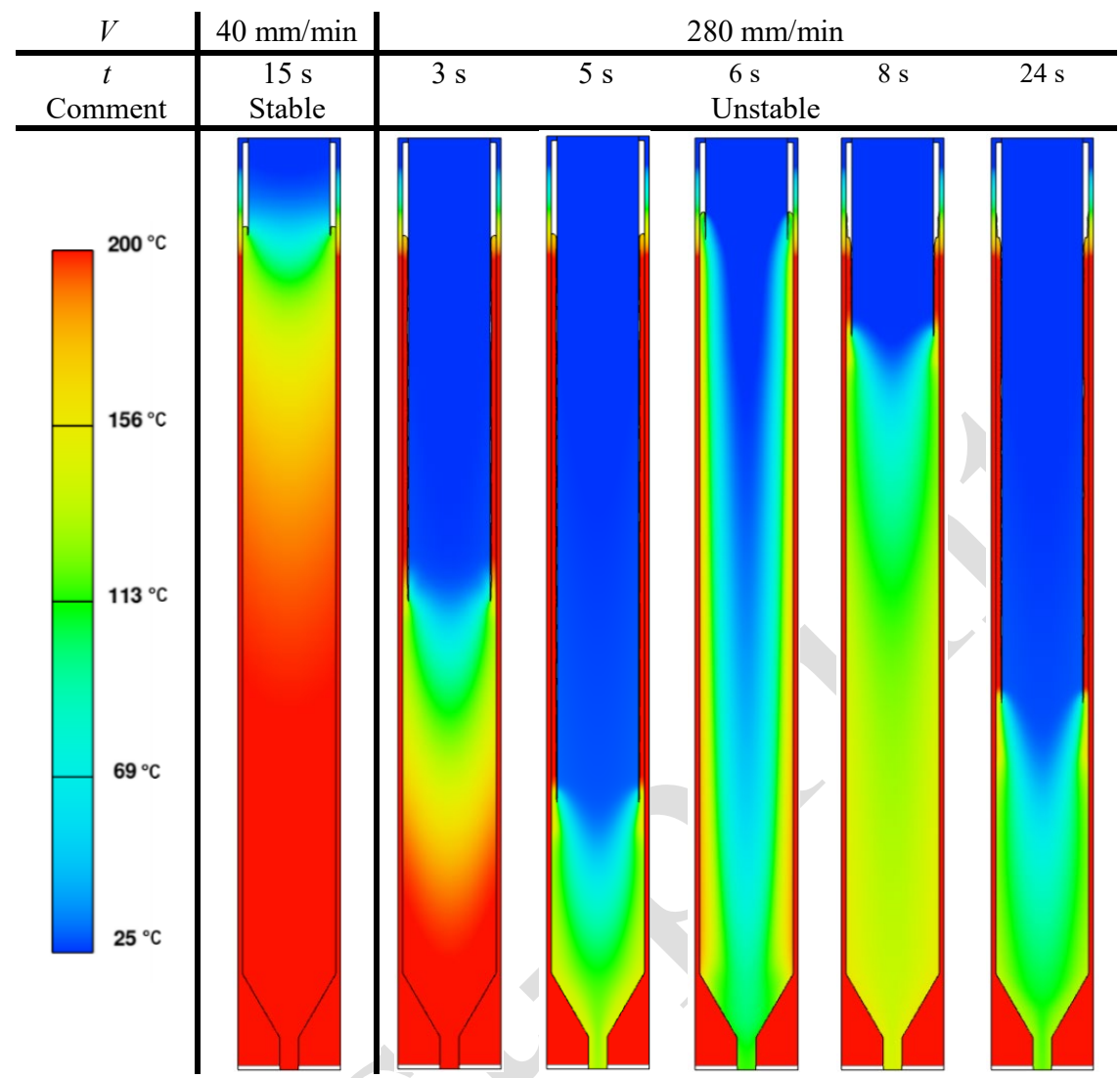

Fig. 7. Evolution of the temperature profiles within the hot-end in stable and unstable extrusion regimes.

\subsection{Influence of the carbon fibers on the flow through the hot-end}

Fig. 8 compares the simulated feeding force as a function of the filament feeding rate for different carbon fiber volume fractions. It can be seen that the increase in the fiber content leads to a higher feeding force, which was expected as the fibers increase the material viscosity. However, at large feeding rates, the feeding force for $\phi=5 \%$ and $\phi=10 \%$ is slightly lower than for a neat polymer $(\phi=0 \%)$. This is explained by the improved thermal conductivity of the filament, which enhances melting of the polymer. The trend is better visible in Fig. 9, where the simulated feeding force was plotted as a function of the fiber volume fraction at different feeding rates. For low and moderate feeding rates, the force increases monotonically with the fiber volume fraction. However, at higher feeding rate, a minimum in the feeding force is observed for $\phi$ from around $5 \%$ to $10 \%$, which indicates that the increased thermal conductivity of the filament can be beneficial for reducing the pressure inside the hot-end.

Except the change in the magnitude of the feeding force, we observe that for high fiber volume fractions, the extrusion remains stable at higher feeding rates. This can be 
seen in Fig. 8 and 9 by the error bars that indicate the force fluctuations, and in Fig. $10 \mathrm{a}$, where the simulated feeding force is shown as a function of time for different fiber volume fractions. The oscillations of the feeding force and the melt zone position determine the maximum feeding rate before the extrusion becomes unstable, as discussed in Section 3.1. The dependence of the maximum feeding rate on the fiber volume fraction, as suggested by the simulations, is shown in Fig. 10b. It is observed that the maximum feeding rate increases linearly as a function of the carbon fiber volume fraction, due to improved effective thermal conductivity of the filament.

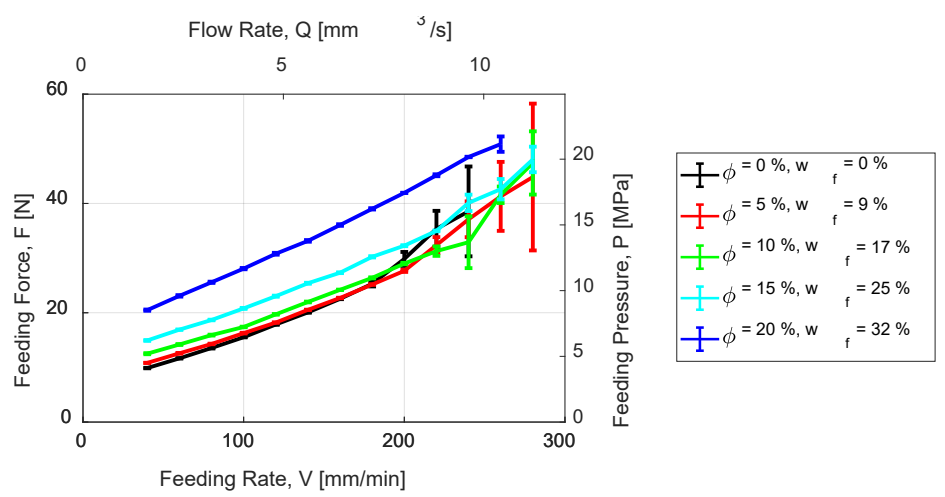

Fig. 8. Feeding force as a function of the filament feeding rate for varying fiber volume fraction. Simulated for $D_{C}=0.4 \mathrm{~mm}$ and $T_{L}=200{ }^{\circ} \mathrm{C}$. The error bars indicate the force oscillations in time.

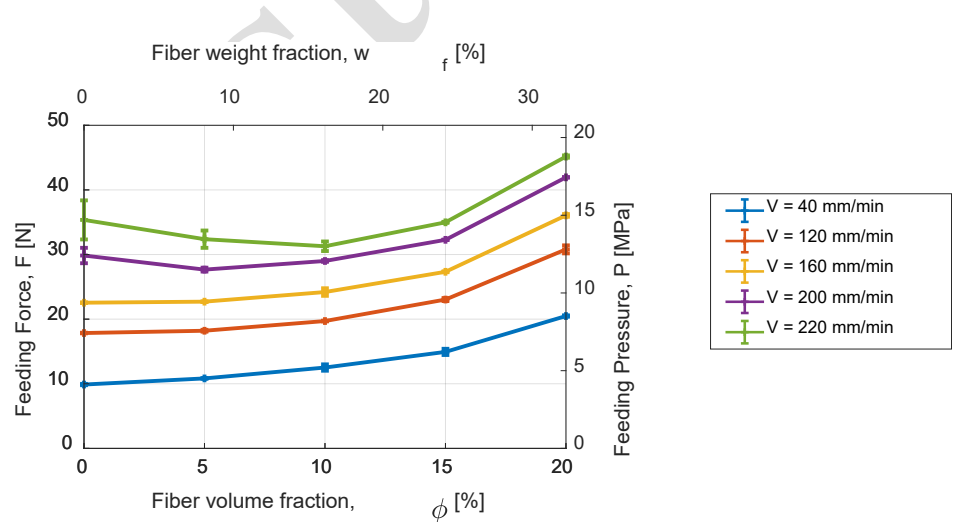

Fig. 9. Feeding force as a function of the fiber volume fraction for varying feeding rates. Simulated for $D_{C}=0.4 \mathrm{~mm}$ and $T_{L}=200^{\circ} \mathrm{C}$. The error bars indicate the force oscillations in time. 

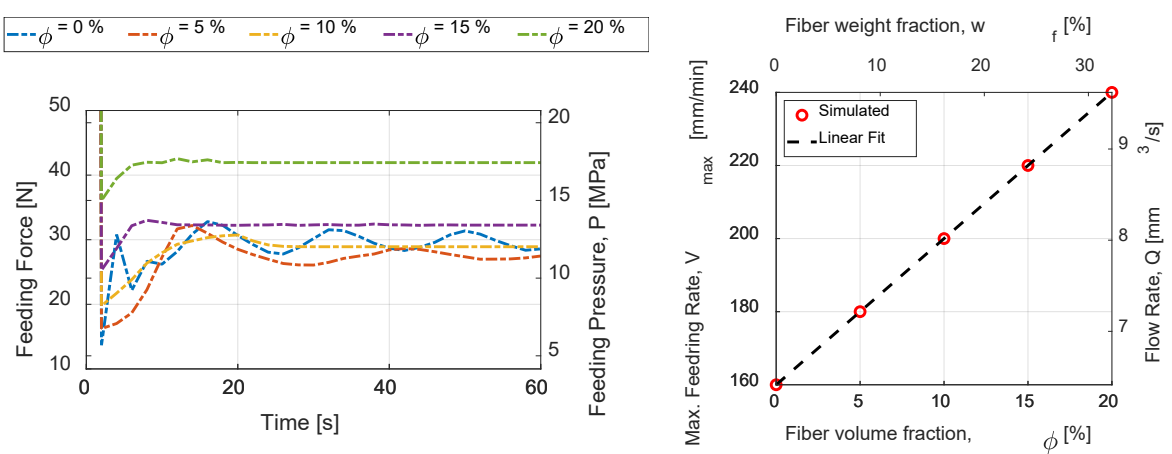

Fig. 10. a) Feeding force as a function of time for different fiber volume fractions, at a feeding rate $\boldsymbol{V}=\mathbf{2 0 0} \mathbf{~ m m} / \mathbf{m i n}$; b) Simulated maximum feeding force (as defined in Section 3.1) as a function of fiber volume fraction.

\section{Conclusions}

This work is one of the first attempts to model the influence of fiber reinforcement on the flow inside the hot-end during material extrusion AM. A CFD model was used to simulate the non-isothermal flow of a power law fluid. The presence of fibers was accounted for by modifying the apparent dynamic viscosity and effective thermal conductivity of the filament. In this work, one of the most commonly used systems was investigated, that is ABS matrix with carbon fibers. However, the model is suitable to simulate fibers made of different materials such as glass, carbon, wood, or nylon. The presented method can be used for short fibers where the aspect ratio (fiber length / fiber diameter) is moderately low (below 25). For long fibers, the elongational viscosity may become more important than shear viscosity. Hence, the Generalized Newtonian Fluid approach may no longer be valid, and more accurate representation of the fluid rheology is required. The results show that the presence of carbon fibers leads to a higher apparent viscosity of the fluid, which, in general, requires a larger feeding force. However, at higher feeding rates, the presence of carbon fibers can be beneficial and decrease the feeding force, because of the increased thermal conductivity that promotes melting of the filament -which ultimately decreases the apparent viscosity. The presented approach could be used to optimize the design of filaments for high printing speed by utilizing fiber reinforcements or particle fillers with high thermal conductivity. The future work shall include experimental measurements in order to confirm the numerical findings. Specifically, the feeding force could be measured for filaments containing varying fiber fractions. 


\section{Acknowledgements}

The authors would like to acknowledge the support of the Danish Council for Independent Research (DFF) | Technology and Production Sciences (FTP) (Contract No. 7017-00128).

\section{Appendix A}

Table 4. Dimensions of the E3D v6 hot-end channel [41].

\begin{tabular}{|l|c|c|c|}
\hline Dimension & Symbol & Unit & Value \\
\hline Nozzle diameter & $D_{C}$ & $\mathrm{~mm}$ & 0.40 \\
\hline Barrel diameter & $D_{B}$ & $\mathrm{~mm}$ & 2.00 \\
\hline Filament diameter & $D_{F}$ & $\mathrm{~mm}$ & 1.75 \\
\hline Capillary length & $L_{C}$ & $\mathrm{~mm}$ & 0.60 \\
\hline Contraction angle & $\alpha$ & ${ }^{\circ}$ & 60 \\
\hline Liquefier section length & $L_{L}$ & $\mathrm{~mm}$ & 15.5 \\
\hline Heat break section length & $L_{H B}$ & $\mathrm{~mm}$ & 2.00 \\
\hline Heat sink section length & $L_{H S}$ & $\mathrm{~mm}$ & 1.00 \\
\hline
\end{tabular}

\section{Appendix B}

The relative viscosity $\eta_{r}$ is the ratio of $\eta_{\phi}$ and $\eta$, defined at the same shear stress

$$
\eta_{r}=\frac{\eta_{\Phi}(\tau)}{\eta(\tau)}=\frac{\tau / \dot{\gamma}_{\phi}}{\tau / \dot{\gamma}}=\frac{\dot{\gamma}}{\dot{\gamma}_{\phi}}
$$

The dynamic viscosity of the filled system can be written as

$$
\eta_{\phi}=\eta \eta_{r}=K \dot{\gamma}^{n-1} \frac{T}{T_{r}} a_{T}^{n} \eta_{r}
$$

where $\dot{\gamma}=\eta_{r} \dot{\gamma}_{\phi}$. Thus, the composite dynamic viscosity is

$$
\eta_{\phi}=K\left(\eta_{r} \dot{\gamma}_{\phi}\right)^{n-1} \frac{T}{T_{r}} a_{T}^{n} \eta_{r}=K \dot{\gamma}_{\phi}^{n-1} \frac{T}{T_{r}} a_{T}^{n} \eta_{r}^{n}
$$




\section{References}

1. Brenken B, Barocio E, Favaloro A, Kunc V, Pipes RB (2018) Fused filament fabrication of fiber-reinforced polymers: A review. Addit Manuf 21:1-16 . https://doi.org/10.1016/j.addma.2018.01.002

2. Hofstätter T, Pedersen DB, Tosello G, Hansen HN (2017) State-of-the-art of fiberreinforced polymers in additive manufacturing technologies. J Reinf Plast Compos 36:1061-1073 . https://doi.org/10.1177/0731684417695648

3. Tekinalp HL, Kunc V, Velez-Garcia GM, Duty CE, Love LJ, Naskar AK, Blue CA, Ozcan S (2014) Highly oriented carbon fiber-polymer composites via additive manufacturing. Compos Sci Technol 105:144-150 https://doi.org/10.1016/j.compscitech.2014.10.009

4. Love LJ, Kunc V, Rios O, Duty CE, Elliott AM, Post BK, Smith RJ, Blue CA (2014) The importance of carbon fiber to polymer additive manufacturing. J Mater Res 29:1893-1898 . https://doi.org/10.1557/jmr.2014.212

5. Brenken B, Barocio E, Favaloro A, Kunc V, Pipes RB (2019) Development and validation of extrusion deposition additive manufacturing process simulations. Addit Manuf 25:218-226 . https://doi.org/10.1016/j.addma.2018.10.041

6. Liaw CY, Guvendiren M (2017) Current and emerging applications of 3D printing in medicine. Biofabrication 9: . https://doi.org/10.1088/1758-5090/aa7279

7. Wohlers Associates (2017) Wohlers Report 2017

8. Goh GD, Agarwala S, Goh GL, Dikshit V, Sing SL, Yeong WY (2017) Additive manufacturing in unmanned aerial vehicles (UAVs): Challenges and potential. Aerosp Sci Technol 63:140-151 . https://doi.org/10.1016/j.ast.2016.12.019

9. Widden M, Gunn K (2010) Design build test of model aerofoils for engineering education using FDM. Virtual Phys Prototyp 5:189-194. https://doi.org/10.1080/17452759.2010.528841

10. Turner BN, Gold SA (2015) A review of melt extrusion additive manufacturing processes: II. Materials, dimensional accuracy, and surface roughness. Rapid Prototyp J 21:250-261 . https://doi.org/10.1108/RPJ-02-2013-0017

11. Šljivi M, Fragassa C, Pavlovi A, Kraišnik M (2016) Additive Manufacturing of Functional Parts Based. 2:178-184 . https://doi.org/10.7251/COMEN1602178S

12. Comminal R, Serdeczny MP, Pedersen DB, Spangenberg J (2018) Numerical modeling of the strand deposition flow in extrusion-based additive manufacturing. Addit Manuf 20:68-76 . https://doi.org/10.1016/j.addma.2017.12.013

13. Serdeczny MP, Comminal R, Pedersen DB, Spangenberg J (2018) Experimental validation of a numerical model for the strand shape in material extrusion additive manufacturing. Addit Manuf 24:145-153 https://doi.org/10.1016/j.addma.2018.09.022

14. Serdeczny MP, Comminal R, Pedersen DB, Spangenberg J (2019) Numerical simulations of the mesostructure formation in material extrusion additive manufacturing. Addit $\quad$ Manuf $28: 419-429$ https://doi.org/10.1016/j.addma.2019.05.024

15. Comminal R, Serdeczny MP, Pedersen DB, Spangenberg J (2019) Motion planning and numerical simulation of material deposition at corners in extrusion additive 
manufacturing. Addit Manuf. https://doi.org/10.1016/j.addma.2019.06.005

16. Xia H, Lu J, Tryggvason G (2018) Fully Resolved Numerical Simulations of Fused Deposition Modeling. Part II-Solidification, Residual Stresses, and Modeling of the Nozzle. Rapid Prototyp J 24:973-987 . https://doi.org/https:// doi.org/10.1108/RPJ-112017-0233

17. Xia H, Lu J, Dabiri S, Tryggvason G (2018) Fully resolved numerical simulations of fused deposition modeling. Part I: fluid flow. Rapid Prototyp J 24:463-476 . https://doi.org/10.1108/RPJ-12-2016-0217

18. Pigeonneau F, D. F, Vincent X, Agassant J-F (2019) Heating and flow computations of an amorphous polymer in the liquefierof a material extrusion 3D printer. Addit Manuf 100820 . https://doi.org/10.1016/j.addma.2019.100820

19. Heller BP, Smith DE, Jack DA (2016) Effects of extrudate swell and nozzle geometry on fiber orientation in Fused Filament Fabrication nozzle flow. Addit Manuf 12:252264 . https://doi.org/10.1016/j.addma.2016.06.005

20. Heller BP, Smith DE, Jack DA (2019) Planar deposition flow modeling of fiber filled composites in large area additive manufacturing. Addit Manuf 25:227-238 . https://doi.org/10.1016/j.addma.2018.10.031

21. Brenken B, Barocio E, Favaloro A, Kunc V, Pipes RB (2019) Development and validation of extrusion deposition additive manufacturing process simulations. Addit Manuf 25:218-226 . https://doi.org/10.1016/j.addma.2018.10.041

22. Favaloro AJ, Brenken B, Barocio E, Pipes RB (2017) Simulation of Polymeric Composites Additive Manufacturing using Abaqus. Sci Age Exp 103-114

23. Go J, Schiffres SN, Stevens AG, Hart AJ (2017) Rate limits of additive manufacturing by fused filament fabrication and guidelines for high-throughput system design. Addit Manuf 16:1-11 . https://doi.org/10.1016/j.addma.2017.03.007

24. Mackay ME, Swain ZR, Banbury CR, Phan DD, Edwards DA (2017) The performance of the hot end in a plasticating 3D printer. J Rheol (N Y N Y) 61:229-236 . https://doi.org/10.1122/1.4973852

25. Nienhaus V, Smith K, Spiehl D, Dörsam E (2019) Investigations on nozzle geometry in fused filament fabrication. Addit Manuf 28:711-718. https://doi.org/10.1016/j.addma.2019.06.019

26. Serdeczny MP, Comminal R, Pedersen DB, Spangenberg J (2020) Experimental and analytical study of the polymer melt flow through the hot-end in material extrusion additive manufacturing. Addit Manuf 32:100997 https://doi.org/10.1016/j.addma.2019.100997

27. Serdeczny MP, Comminal R, Mollah MT, Pedersen DB, Spangenberg J Numerical modeling of the polymer flow in the hot-end of material extrusion additive manufacturing. Addit Manuf (In Press

28. Bird, R. B., Armstrong RC, Hassager O (1987) Dynamics of Polymeric Liquids. JOHN WILEY \& SONS

29. FLOW-3D | We Solve the World's Toughest CFD Problems. https://www.flow3d.com/. Accessed 4 Sep 2019

30. Kitano T, Kataoka T, Shirota T (1981) An empirical equation of the relative viscosity of polymer melts filled with various inorganic fillers. Rheol Acta 20:207-209 . https://doi.org/10.1007/BF01513064 
31. Metzner AB (1985) Rheology of Suspensions in Polymeric Liquids. J Rheol (N Y N Y) 29:739-775 . https://doi.org/10.1122/1.549808

32. Ning F, Cong W, Qiu J, Wei J, Wang S (2015) Additive manufacturing of carbon fiber reinforced thermoplastic composites using fused deposition modeling. Compos Part B Eng 80:369-378 . https://doi.org/10.1016/j.compositesb.2015.06.013

33. Jeon YP, Alway-Cooper R, Morales M, Ogale AA (2013) Carbon Fibers, Second Edi. Elsevier

34. Fu SY, Lauke B, Mäder E, Yue CY, Hu X, Mai YW (2001) Hybrid effects on tensile properties of hybrid short-glass-fiber-and short-carbon-fiber-reinforced polypropylene composites. J Mater Sci 36:1243-1250 . https://doi.org/10.1023/A:1004802530253

35. Vaxman A, Narkis M, Siegmann A, Kenig S (1989) Short-fiber-reinforced thermoplastics. Part III: Effect of fiber length on rheological properties and fiber orientation. Polym Compos 10:454-462 . https://doi.org/10.1002/pc.750100610

36. Guo R, Azaiez J, Bellehumeur C (2005) Rheology of fiber filled polymer melts: Role of fiber-fiber interactions and polymer-fiber coupling. Polym Eng Sci 45:385-399 . https://doi.org/10.1002/pen.20285

37. Fu S, Mai Y (2003) Thermal Conductivity of Misaligned Short-Fiber- Reinforced Polymer Composites. J Appl Polym Sci 88:1497-1505 . https://doi.org/10.5772/66336

38. Osswald TA, Puentes J, Kattinger J (2018) Fused filament fabrication melting model. Addit Manuf 22:51-59 . https://doi.org/10.1016/j.addma.2018.04.030

39. Carbon UD HS 194 gsm. www.tohotenax-eu.com. Accessed 31 Jan 2020

40. Serdeczny MP, Comminal R, Pedersen DB, Spangenberg J (2019) Experimental analysis of the filament feeding force in material extrusion additive manufacturing. Proc Jt Spec Interes Gr Meet between euspen ASPE Adv Precis Addit Manuf 141-145

41. E3D-Online Documentation - E3D-Online. https://e3d-online.dozuki.com/. Accessed 3 Jul 2019 